\title{
ADESÃO DE PACIENTES COM AIDS AO TRATAMENTO COM ANTIRETROVIRAIS: DIFICULDADES RELATADAS E PROPOSIÇÃO DE MEDIDAS ATENUANTES EM UM HOSPITAL ESCOLA
}

\author{
Rosely Moralez de Figueiredo ${ }^{1}$ \\ Verônica Maria Sinkoc ${ }^{2}$ \\ Cybele Cristina Tomazim ${ }^{3}$ \\ Maria Cecilia Bueno Jayme Gallani ${ }^{4}$ \\ Maria Rosa Ceccato Colombrini ${ }^{5}$
}

Figueiredo RM, Sinkoc VM, Tomazim CC, Gallani MCBJ, Colombrini MRC. Adesão de pacientes com AIDS ao tratamento com antiretrovirais: dificuldades relatadas e proposição de medidas atenuantes em um hospital escola. Rev Latino-am Enfermagem 2001 julho; 9(4):50-5.

Com finalidade de propor medidas que facilitem a adesão do paciente com AIDS ao tratamento, este trabalho teve como objetivo caracterizar os erros e as principais dificuldades relatadas por 61 pacientes com AIDS atendidos junto ao Hospital de Clínicas da UNICAMP. Os erros mais freqüentes foram referentes às combinações entre as drogas e falta de jejum; as dificuldades relatadas foram: quantidade de medicamentos e reações adversas. As autoras propuseram, a partir destes dados, a utilização de um roteiro ilustrado para realização de orientações relativas aos medicamentos. Espera-se assim que a orientação individualizada possa contribuir para minimizar as falhas da adesão à terapêutica.

PALAVRAS CHAVE: aderências, terapêutica, AIDS, educação em saúde

\section{ADHERENCE OF AIDS PATIENTS TO TREATMENT WITH ANTIRETROVIRAL DRUGS IN A UNIVERSITY HOSPITAL: DIFFICULTIES OBSERVED AND SUGGESTIONS OF INTERVENTIONS}

This study aimed at identifying the incidence of drug intake errors and the main therapy-related difficulties among AIDS patients as well as at proposing measures to increase patients' adherence to treatment. Sixty-one patients with AIDS assisted by the University Hospital of São Paulo State University at Campinas were interviewed. The most frequent errors concerned the combinations of drugs and the length of fasting. The difficulties reported were adverse effects and the variety of drugs. Based on these data, the authors proposed the use of an illustrated guide for drug-related information. It is hoped that individualized guidance can contribute to minimize errors concerning adherence to therapy.

KEY WORDS: adherence, therapeutics, AIDS, health education

\section{ADHESIÓN DEL PACIENTE CON SIDA AL TRATAMIENTO CON ANTIRETROVIRALES: DIFICULTADES RELATADAS Y PROPUESTA DE MEDIDAS ATENUANTES EN UN HOSPITAL ESCUELA}

Con la finalidad de proponer medidas que faciliten la adhesión del paciente con SIDA al tratamiento, este trabajo tuvo como objetivo caracterizar los errores y las principales dificultades relatadas por 61 pacientes con SIDA atendidos en el Hospital Clínicas de la UNICAMP. LOS errores más frecuentes estuvieron relacionados con las combinaciones entre las drogas y la falta de ayuno; las dificultades relatadas fueron: Cantidad de medicamentos y reacciones adversas. Las autoras propusieron, a partir de estos datos, la utilización de una guía ilustrada para dar orientaciones relacionadas con los medicamentos. Se espera que la orientación individualizada pueda contribuir para disminuir las fallas en la adhesión al tratamiento.

PALABRAS CLAVES: adhesión, tratamiento, SIDA, educación en salud

\footnotetext{
${ }^{1}$ Mestre e Doutor em Saúde Mental. Enfermeira NVE/HC - UNICAMP e Professor das Faculdades Pe Anchieta de Jundiaí. End. Universidade Estadual de Campinas (UNICAMP), Hospital de Clínicas/NVE, e-mail: rosely@hc.unicamp.br; ${ }^{2}$ Mestranda em Saúde Coletiva, Enfermeira NVE/HC - UNICAMP, ${ }^{3}$ Graduanda em Enfermagem FCM - UNICAMP; ${ }^{4}$ Professor Colaborador do Depto Enfermagem - FCM - UNICAMP, Doutor em Enfermagem; ${ }^{5}$ Enfermeira diretora do SEMC II - HC - UNICAMP e mestranda em Enfermagem/UNICAMP
} 


\section{INTRODUÇÃO}

Com o advento dos antiretrovirais no tratamento da AIDS observou-se uma melhora significativa na qualidade de vida dos pacientes $^{(1-2)}$. Com isto a AIDS passa a ter características de uma doença crônica e apesar do grande benefício gerado por esta terapêutica, restam ainda muitas dificuldades a serem superadas. Uma delas é a adesão do paciente ao seu tratamento ${ }^{(3)}$.

Fatores como a quantidade de medicamentos, as reações adversas (intolerância), a necessidade de períodos de jejum, a incompatibilidade entre as drogas, a dificuldade na compreensão das metas da terapia e da implicação do seu uso inadequado, contribuem para dificultar o processo terapêutico ${ }^{(3-7)}$.

Vários autores ${ }^{(6,8)}$ referem que, em geral, as taxas de adesão para tratamento de doenças crônicas, com tratamento prolongado já são baixas, e isto se agrava ainda mais quando se associa a uma doença com limitada perspectiva de sobrevida. Os autores categorizam os fatores relacionados à adesão de acordo com características do paciente, do regime de tratamento, do vínculo com os profissionais, da inserção social e severidade da doença.

Pode-se considerar ainda a complexidade posológica e a adesão do paciente ao tratamento por períodos prolongados como um desafio à efetividade dos recursos terapêuticos disponíveis contra a $\operatorname{AIDS}^{(3-4)}$.

Esta situação, além de não propiciar o efeito terapêutico desejado, pode contribuir para o desenvolvimento de resistência viral às drogas utilizadas. $O$ desenvolvimento de resistência e a subseqüente perda da ação das drogas contra o HIV constituem a barreira primária para a eficácia do tratamento a longo prazo. Há que se considerar ainda a habilidade do vírus produzir mais cópias de si mesmo, e ao fazer isto, gerar mutações espontâneas do genoma viral $^{(9-11)}$

Pesquisadores sugerem diferentes formas de se avaliar 0 seguimento do tratamento prescrito, sendo uma delas a quantificação dos níveis séricos da droga. Entretanto, por serem drogas que possuem meia-vida plasmática curta, este dado é verificado somente se os pacientes tiverem feito uso recente da última dose, isto sem mencionar o custo envolvido no processo. A avaliação empírica do médico, sem a coleta de informações adicionais específicas, tende a ser baseada em noções preconcebidas, freqüentemente enganosas, de acordo com as quais os pacientes seriam aderentes. $O$ auto-relato do paciente, segundo 0 autor, quando feito adequadamente, seria uma ótima maneira de se avaliar a adesão na prática clínica, considerando-o, inclusive, mais preciso de que a avaliação médica ${ }^{(12)}$.

A realização de perguntas específicas, sem julgamento, poderia por sua vez, maximizar a oportunidade de obter informações úteis para a adesão ao tratamento ${ }^{12}$. Neste contexto, a interação pessoal parece mostrar-se como uma ferramenta barata e eficaz para a otimização desta intervenção. Entretanto, em instituições como hospitais escolas, onde há uma grande rotatividade de pessoal envolvido na assistência, existe uma certa dificuldade em se conseguir manter a padronização das informações fornecidas e a estabilidade da relação profissional de saúde-paciente, condições críticas para início e manutenção de uma intervenção pessoal efetiva ${ }^{(13)}$.

A enfermeira mostra-se ser o profissional mais familiarizado com o problema e o desafio da adesão, uma vez que, de um modo geral, já possui ampla experiência na abordagem a pacientes com diabetes mellitus, hipertensão arterial ou em uso de terapêutica psiquiátrica, sendo por conseguinte, um profissional adequado para esta intervenção ${ }^{(6)}$.

Na prática assistencial junto a pacientes que fazem uso de antiretrovirais, tem sido observado com frequêencia, que estes apresentam queixas e dificuldades de compreensão no uso dos esquemas terapêuticos. Considerando que tais fatores podem ser críticos no comprometimento da adesão do paciente à terapêutica medicamentosa, este trabalho teve como objetivo caracterizar os erros e as principais dificuldades relatadas para a utilização da terapêutica antiretroviral por pacientes soropositivos para HIVIAIDS em tratamento ambulatorial e na unidade leito-dia do Hospital de Clínicas (HC) da Universidade Estadual de Campinas (UNICAMP).

Pretende-se, a partir do resultado deste trabalho, desenvolver e implementar intervenções que auxiliem o paciente e 0 cuidador na adesão à terapêutica medicamentosa.

\section{PROCEDIMENTOS METODOLÓGICOS}

O estudo foi realizado junto ao Ambulatório de Doenças Sexualmente Transmissíveis/AIDS e à Unidade Leito-Dia em AIDS do HC- UNICAMP.

Este hospital é referência para atendimento a pessoas portadoras da AIDS na cidade de Campinas e região que englobam uma população de 5 milhões de habitantes, e contava com aproximadamente 600 pacientes com HIVIAIDS em seguimento no serviço no ano em questão.

Foram entrevistados 61 pacientes, compondo uma amostra de aproximadamente $10 \%$ dos pacientes adultos soropositivos para HIVIAIDS em tratamento no Hospital de Clínicas da Universidade de Campinas no ano de 1997.

Foram selecionados os primeiros 61 pacientes que faziam uso de algum antiretroviral e que compareceram à Unidade LeitoDia ou ao Ambulatório, no mês de outubro de 1997.

Uma vez contatado, o paciente era informado quanto à pesquisa, sua finalidade e procedimento da entrevista, além de ser- 
Ihe assegurado que a participação dele na pesquisa não iria interferir em nenhum aspecto do seu tratamento. Em seguida, era obtido 0 consentimento do paciente para a participação no estudo e o mesmo conduzido a uma sala privativa para realização da entrevista.

Foi construído um instrumento (Anexo 1) composto por 12 questões, abertas e fechadas, direcionadas para o levantamento dos seguintes dados: caracterização do paciente e das medicações em uso: tipo, horário, cuidados especiais adotados pelo paciente, bem como, fatores que facilitavam e que dificultavam seu uso adequado.

Foi utilizado software EPI-INFO para o registro dos dados a serem analisados. Aplicou-se $o$ teste $t$ de Student para a comparação das médias de variáveis quantitativas e o teste QuiQuadrado $\left(c^{2}\right)$ para verificar a associação entre algumas variáveis categóricas.

\section{RESULTADOS E DISCUSSÃO}

Dos 61 pacientes entrevistados, $41(67,2 \%)$ eram do sexo masculino, 38 (62,3\%) não trabalhavam e $20(32,8 \%)$ precisavam de ajuda para tomar seus remédios.

Mais de $60 \%$ dos pacientes utilizavam-se, além do nome, do formato dos comprimidos para identificá-los (Tabela 1).

Tabela 1 - Distribuição da freqüência dos recursos utilizados para identificação dos medicamentos, de acordo com o relato dos 61 pacientes. HC-UNICAMP. Campinas, 1997

\begin{tabular}{lcc}
\hline \multicolumn{1}{r}{ Recursos } & N & \% \\
\hline Nome & 42 & 68,8 \\
Formato & 38 & 62,3 \\
Cor & 15 & 24,6 \\
Horário de ingestầo & 11 & 18,0 \\
\hline
\end{tabular}

Obs. Cada entrevistado pode apontar mais de um recurso

Quanto às dificuldades, 29 (47,5\%) dos pacientes referiramse aos efeitos colaterais e $16(26,2 \%)$ ao volume de medicamentos (Tabela 2).

Tabela 2 - Distribuição da freqüência das dificuldades para a ingestão dos medicamentos, de acordo com o relato dos 61 pacientes. HCUNICAMP. Campinas, 1997

\begin{tabular}{lcc}
\hline \multicolumn{1}{c}{ Dificuldades } & N & $\%$ \\
\hline Efeitos colaterais & 29 & 47,5 \\
Wolume de comprimidos & 16 & 26,2 \\
Horánios & 14 & 22,9 \\
Sabor & 06 & 9,8 \\
\hline
\end{tabular}

Obs. Cada entrevistado pode relatar mais de uma dificuldade
Os erros mais freqüentes estiveram relacionados ao uso combinado de drogas, relatado por 16 pacientes $(26,2 \%)$ e à falta de jejum, citada por $13(21,3 \%)$ dos pacientes. As drogas mais associadas aos erros foram a Didadosina e o Indinavir, como constatado em $12(19,7 \%)$ e $16(26,2 \%)$ das respostas, respectivamente (Tabela 3).

Tabela 3 - Distribuição da freqüência dos tipos de erros cometidos na utilização dos antiretrovirais, de acordo com o relato dos 61 pacientes. HC-UNICAMP. Campinas, 1997

\begin{tabular}{lcc}
\hline \multicolumn{1}{c}{ Tipo de erro } & N & $\%$ \\
\hline Associaç̃̃o medicamentosa & 16 & 26,2 \\
Relacionado ao indinguir & 16 & 26,2 \\
Nẫo cumprimento de jejum & 13 & 21,3 \\
Relacionado à Didadosing & 12 & 19,7 \\
\hline
\end{tabular}

Obs. Cada entrevistado pode responder a mais de uma alternativa

Quanto ao grau de escolaridade, foi observado que 0 número de anos de estudo oscilou de um a 15 anos (Tabela 4). Constatou-se também que o número de anos de estudo esteve associado ao erro/acerto no emprego da prescrição médica, de forma que, no grupo onde houve acerto, a média de anos de estudo foi de 8,4 anos, significativamente maior que a do grupo onde houve erro, que foi de 6,6 anos $(p<0,05)$.

Tabela 4 - Distribuição da freqüência dos 61 pacientes de acordo com erro ou acerto no emprego da prescrição médica e o nível de escolaridade, dado pelo número de anos de estudo. HC-UNICAMP. Campinas, 1997

\begin{tabular}{|c|c|c|c|c|c|c|}
\hline \multirow[t]{2}{*}{$\begin{array}{l}N^{0} \text { de anos } \\
\text { de estudo }\end{array}$} & \multicolumn{4}{|c|}{$\begin{array}{l}\text { Erro no emprego Acerto no emprego } \\
\text { da prescriģa da prescrição }\end{array}$} & \multicolumn{2}{|c|}{ TOTŔL } \\
\hline & N & $\%$ & N & $\%$ & N & $\%$ \\
\hline 0 a 4 & 09 & 14,8 & 07 & 11,5 & 16 & 26,3 \\
\hline $5 a$ & 10 & 16,4 & 12 & 19 & 22 & 36,1 \\
\hline 9 ou mais & 06 & 9,8 & 17 & 27,8 & 23 & 37,6 \\
\hline TOTÁL & 25 & 41,0 & 36 & 59,0 & 61 & 100,0 \\
\hline
\end{tabular}

O número, por paciente, de medicamentos utilizados variou de dois a 13 e também guardou associação com a obediência à prescrição (Tabela 5).

Tabela 5 - Distribuição da freqüência dos 61 pacientes de acordo com o erro ou o acerto no emprego da prescrição médica e o número de medicações prescritas. HC-UNICAMP. Campinas, 1997

\begin{tabular}{|c|c|c|c|c|}
\hline \multirow{2}{*}{$\begin{array}{c}\text { Húmero de } \\
\text { medicaçóes } \\
\text { prescritas }\end{array}$} & \multicolumn{2}{|c|}{$\begin{array}{l}\text { Erro no seguimento da } \\
\text { prescrição }\end{array}$} & \multicolumn{2}{|c|}{$\begin{array}{l}\text { Acerto no seguimento } \\
\text { da prescriģão }\end{array}$} \\
\hline & N & $\%$ & $\mathbf{H}$ & $\%$ \\
\hline $\begin{array}{c}1 \text { a } 3 \\
4 \text { a } 6 \\
7 \text { a } 9 \\
10 \text { ou mais } \\
\text { TOTaL }\end{array}$ & $\begin{array}{l}02 \\
11 \\
10 \\
02 \\
\mathbf{2 5}\end{array}$ & $\begin{array}{c}3,3 \\
18,0 \\
16,4 \\
3,3 \\
\mathbf{4 1 , 0}\end{array}$ & $\begin{array}{l}13 \\
17 \\
05 \\
01 \\
\mathbf{3 6}\end{array}$ & $\begin{array}{c}21,3 \\
27,9 \\
8,2 \\
1,6 \\
59,0\end{array}$ \\
\hline
\end{tabular}


Vinte e cinco pacientes faziam uso inadequado da medicação, sendo que destes, 15 (24,5\%) acreditavam estar tomando corretamente os medicamentos, quando na verdade sua prática era diferente da recomendada na prescrição (Tabela 6). Estes achados reafirmam a existência de falhas no seguimento à terapêutica proposta aos pacientes seguidos no HC-UNICAMP.

Tabela 6 - Distribuição dos 61 pacientes de acordo com o erro e ou acerto no seguimento da prescrição médica e a sua opinião quanto ao uso adequado ou não dos medicamentos. HC-UNICAMP. Campinas, 1997

\begin{tabular}{|c|c|c|c|c|}
\hline \multirow[t]{2}{*}{ Opiniäo do paciente } & \multicolumn{2}{|c|}{$\begin{array}{l}\text { Erro no seguimento } \\
\text { da prescriģão }\end{array}$} & \multicolumn{2}{|c|}{$\begin{array}{l}\text { Acerto no seguimento } \\
\text { da prescrição }\end{array}$} \\
\hline & $\mathbf{H}$ & $\%$ & $\mathbf{H}$ & $\%$ \\
\hline $\begin{array}{l}\text { "Acho que faço uso ade- } \\
\text { quado dos medic amen- } \\
\text { tos" }\end{array}$ & 15 & 60,0 & 36 & 83,3 \\
\hline $\begin{array}{l}\text { "A Acho que não faço uso } \\
\text { adequado dos medica- } \\
\text { mentos" }\end{array}$ & 10 & 40,0 & 06 & 16,7 \\
\hline TOTÁL & 25 & 100,0 & 42 & 100,0 \\
\hline
\end{tabular}

$x^{2}=4,15 p>0,05$

$\mathrm{Na}$ tentativa de minimizar estes erros foram elaboradas duas formas de intervenção: um quadro ilustrativo e um roteiro de medicações.

Quanto ao quadro ilustrativo, o mesmo foi confeccionado com as embalagens, comprimidos e cuidados necessários com cada droga e fixado nas unidades (ambulatórios e Leito-dia) para ser utilizado como roteiro ilustrativo na atividade de orientação (Anexo 2). Desta maneira, sempre que o paciente receber sua receita pela primeira vez ou a mesma for alterada, a enfermeira orientará 0 paciente utilizando-se desta ilustração, de forma que além do nome, o paciente poderá visualizar a embalagem e o formato do comprimido ou cápsula que deverá ingerir.

Para facilitar a compreensão do paciente e cuidador de como organizar seu dia, levando em consideração a rotina de cada paciente, a enfermeira da unidade deve elaborar um roteiro segundo a prescrição médica, onde os medicamentos são associados aos horários de jejum, refeições, trabalho e sono (Anexo 3).

Pesquisadores consideram a atenção individualizada a grande aliada da melhora da adesão e a relação enfermeira-paciente a sua ferramenta mais importante para sua efetivação ${ }^{(1,3)}$.
As autoras ${ }^{(1,3)}$ comentam também que a confiança é o elemento chave desta relação e para que ela ocorra é necessário o estabelecimento de empatia, de credibilidade no profissional junto ao grupo, do respeito à privacidade e, principalmente, de confiança nas informações e nos comportamentos do paciente.

Neste processo educativo são atitudes positivas: determinação de metas com flexibilidade, utilização de termos curtos, a consideração do contexto social onde está inserido o paciente e a não realização de julgamentos morais. A utilização de recursos didáticos como panfletos e guias são de excelente ajuda, mas não substituem a atenção individual do profissional ${ }^{(3)}$.

Soluções formuladas em conjunto (enfermeira/paciente) tendem a ser muito mais respeitadas e há um empenho maior para que elas dêem certo ao vez de respostas prontas, pré-fixadas ${ }^{(1)}$.

\section{CONSIDERAÇÕES FINAIS E SUGESTÕES}

Os dados obtidos neste trabalho apontaram para a existência de dificuldades e erros na utilização do complexo esquema da terapêutica medicamentosa pelos pacientes soropositivos para HIVIAIDS atendidos ambulatorialmente e na Unidade Leito-Dia em AIDS na UNICAMP.

Este grupo de pacientes foi caracterizado por ser constituído predominantemente pelo sexo masculino, por pessoas profissionalmente inativas, com a mediana de escolaridade de 7 anos de estudo e que relataram dificuldades para o uso dos medicamentos. Tais dificuldades foram relativas ao efeito colateral das drogas, grande volume dos comprimidos, horários de utilização e sabor desagradável. O grupo apresentou também erros no que se refere a associação inadequada de medicamentos, não cumprimento do jejum e no uso específico de alguns antiretrovirais (Indinavir e Didadosina). Constatou-se que o nível de escolaridade e o número de medicamentos empregados por paciente estiveram associados com a adesão à terapêutica. 0 grupo pesquisado mostrou lançar mão de alguns meios para facilitar a utilização dos medicamentos.

Dessa forma, tais resultados apontam para a clara necessidade do desenvolvimento e implementação de intervenções que sejam realisticamente desenhadas para grupos específicos visando facilitar a adesão do paciente à terapia medicamentosa. 


\section{Anexo 1}

\section{CARACTERIZAÇÃO DAS DIFICULDADES DE ADESÃO DE PACIENTES COM AIDS AO TRATAMENTO MEDICAMENTOSO VIA ORAL}
1) Nome
H.C.
2) Idade
Sexo
3) Escolaridade
Tempo de tratamento
4) Local da coleta
Atividade ocupacional ( ) S ( ) N
5) Acuidade visual - ( ) nega queixas ( ) refere déficit ( ) déficit diagnosticado
6) Medicações V.O. em uso:

\begin{tabular}{|l|l|l|l|l|l|}
\hline Antivirais & Proftstat.PPC & Proftsrt.TB & Proftrat.toxo & Profftat.cript & Outros \\
\hline & & & & & \\
\hline & & & & & \\
\hline
\end{tabular}

7) Quais os horários dos seus remédios e cuidados especiais (jejuns e combinações)

8) Você toma seus remédios...:

( ) Sozinho ( ) Com ajuda? (de quem?) ( ) Alguém dá para você? 9) Como você faz para reconhecer seus medicamentos? (nome, cor, tamanho, formato, etc)

10) Você sente dificuldades em tomar os remédios?

Quais? (quantidade, horários, jejum, efeitos colaterais, receitas, tempo de tratamento, etc)

11) Você entende a receita do médico? ( ) Sim ( ) Não ( ) Parcialmente

12) Você toma seus medicamentos exatamente como sua receita?
( ) Sim
( ) Não. Por que?

\section{Anexo 2}

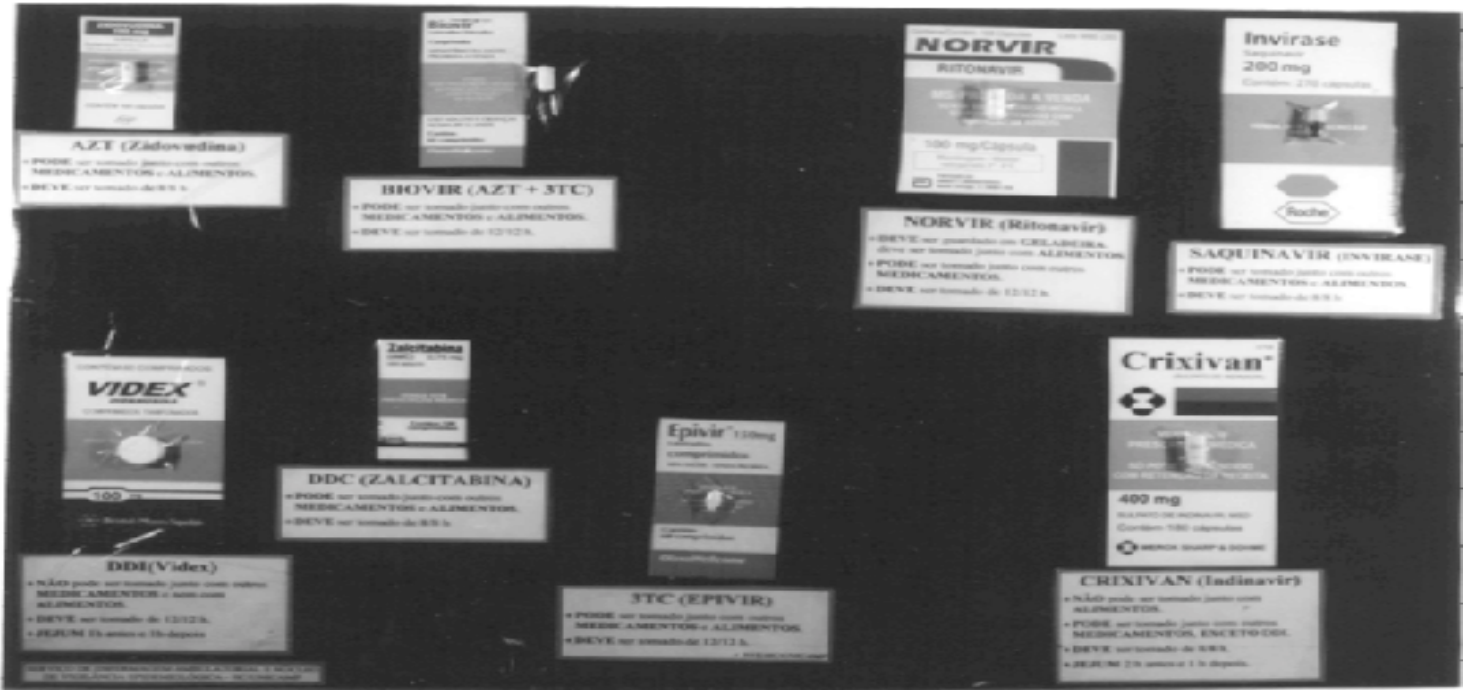

Anexo 3

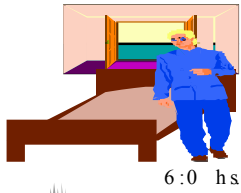

Crixiva-2

AZT-com

Sulfadiazin- $3 \mathrm{com}$

Pirimetam $i-2$ com

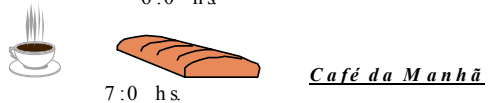

$7: 0 \mathrm{~h}$

Café da Manhã
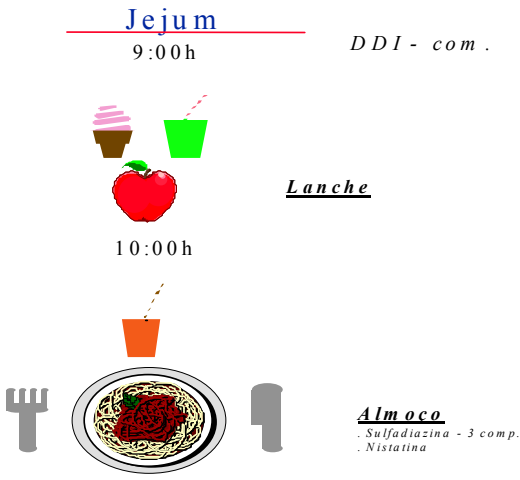

$12: 00 \mathrm{~h}$

$14: 00 \mathrm{~h}$

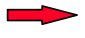

Crixiva $-2 \mathrm{com}$

Roteiro com Exemplo de Preenchimento

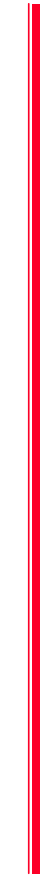

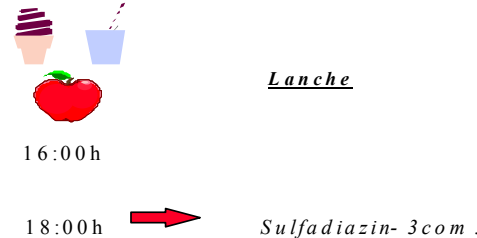

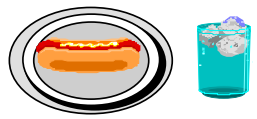

$\underline{\text { Jantar }}$

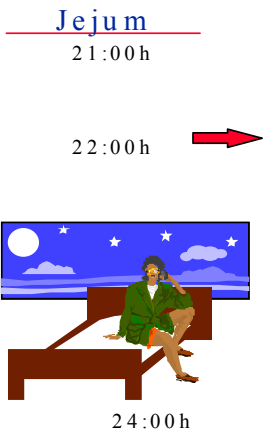

$D D I-\operatorname{com}$

$A Z T-3 c o m$

Crixtatin

Sulfadiazin- $3 \mathrm{com}$ 


\section{REFERÊNCIAS BIBLIOGRÁFICAS}

1. Tourete-Turgis C. Infection à V.I.H. trithérapies: guide counseling. Paris: Mâitre de Conférence des Universités Co-foundatrice de Comment Dire; 1997.

2. Baker S. Home Care: adressing the needs of people living with AIDS and their caregivers. Nurs Clin North Am 1999 Mar; 34(1):20112.

3. Crespo-Fierro M. Compliance/adherence and care management in HIV disease. J Assoc Nurses AIDS Care 1997 July/August; 8(4):4354.

4. Figueiredo RM, Sinkoc VM, Gallani MBJ, Tomazin C, Cavinatto SM, Assumpção ISA et al. Adherence of pacients with AIDS to treatment with antiretroviral medications: dificulties related and proposition of attenuating measures. Proceedings of the $12^{\text {th }}$ World AIDS Conference; 1998 June-July; Geneva: Conference Record 1998. p.862.

5 - THERREN, R; GAGNON, A; MAILHOT, M. Increasing patient adherence: experience with the "HIV medication guide". Proceedings of the $12^{\text {th }}$ World AIDS Conference, 1998.june-july; Geneva: Conference Record; 1998. p.528.

6. Williams AB. Adherence to highly active antiretroviral teraphy. Nurs Clin North Am 1999 Mar; 34(1):113-9.
7. Sinkoc VM, Figueiredo RM, Colombrini MRC, Cavinato SM, Tomazin

C, Gallani MCJ. Dificuldades referidas por pacientes com AIDS na adesão ao tratamento com antiretrovirais. Braz J Infect Dis 1999 August; 3(Suppl):S60.

8. Ickovics JR, Meisler AW. Adherence in AIDS clinical trials: a framework for clinical research and clinical care. J Clin Epidemiol 1997; 50(4):385-91.

9. Davey RT Jr. The changing face of antiretroviral therapeutics in 1997. Home Healthcare Consultant 1997 September; 4(8):10-28.

10 Molaghan JB. Adherence issues in HIV therapeutics introduction: the situation. J Assoc Nurses AIDS Care 1997; 8(Suppl):7-9.

11. Unguarski P. Adherence to prescribe HIV-1 protease inhibitors in the home setting. J Assoc Nurses AIDS Care 1997; 8(Suppl): 37-45. 12. Hechet FM. Avaliação da aderência ao tratamento do HIV na prática clínica. AIDS Clinical Care 1998 Julho/Agosto; 1(1):1-4.

13. Camargo AMA, Assumpção ISMA, Cavinatto SM, Sinkoc VM, Pimenta NM. Integral treatment of tuberculosis patients. Proceedings of the Conference on Global Lung Health and Annual Meeting of the International Union Against Tuberculosis and Lung Disease; 1997

October 1-4; Paris: International Journal of Tuberculosis and Lung Disease; 1997. 